\title{
OCEAN INFORMATION PROVIDED THROUGH ENSEMBLE OCEAN SYNTHESES
}

D. Stammer ${ }^{(1)}$, A. Köhl ${ }^{(1)}$, T. Awaji ${ }^{(2)}$, M. Balmaseda ${ }^{(3)}$, D. Behringer ${ }^{(4)}$, J. Carton $^{(5)}$, N. Ferry ${ }^{(6)}$ A. Fischer $^{(7)}$, I. Fukumori $^{(8)}$, B. Giese ${ }^{(9)}$, K. Haines ${ }^{(10)}$, E. Harrison ${ }^{(11)}$, P. Heimbach ${ }^{(12)}$, M. Kamachi ${ }^{(13)}$, C. Keppenne ${ }^{(14)}$, T. Lee $^{(8)}$, S. Masina ${ }^{(15)}$, D. Menemenlis ${ }^{(8)}$, R. Ponte ${ }^{(16)}$, E. Remy $^{(6)}$, M. Rienecker $^{(14)}$, A. Rosati ${ }^{(17)}$, J. Schröter ${ }^{(18)}$,

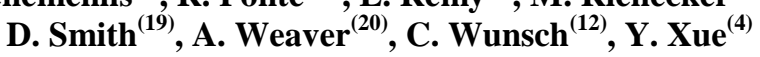

(1) Institut für Meereskunde, KlimaCampus, University of Hamburg, Bundesstr. 53, 20146 Hamburg, Germany, Email: detlef.stammer@zmaw.de; armin.koehl@zmaw.de

${ }^{(2)}$ Faculty of Science, Kyoto University, Sakyo-ku, Kyoto 606-01 - Japan, awaji@kugi.kyoto-u.ac.jp

(3) ECMWF (European Centre for Medium-Range Weather Forecasts), Shinfield Park, Reading, RG2 9AX, United Kingdom, Email: Magdalena.Balmaseda@ecmwf.int

(4) Climate Prediction Center, NCEP/NOAA (/National Centers for Environmental Prediction /National Oceanic and Atmospheric Administration), 5200 Auth Road, Room 605, Camp Springs, MD 20746 USA, Email: david.behringer@noaa.gov; Yan.Xue@noaa.gov

${ }^{(5)}$ Department of Atmospheric and Oceanic Science, 3413 Computer \& Spaces Sci. Bldg., Univ. MD., College Park, MD 20742 USA, Email: carton@atmos.umd.edu

${ }^{(6)}$ Mercator-Océan, 8-10 rue Hermès, 31520 Ramonville Saint-Agne, France, Email: nicolas.ferry@mercator-ocean.fr

(7) Intergovernmental Oceanographic Commission, United Nations Educational, Scientific and Cultural Organization -

1 rue Miollis - 75732 Paris Cedex 15 - France Email: a.fischer@unesco.org

${ }^{(8)}$ NASA (National Aeronautics and Space Administration) Jet Propulsion Laboratory, California Institute of Technology, 4800 Oak Grove Dr., Pasadena, CA 91109 USA, Email: tlee@jpl.nasa.gov

(9) Dept of Oceanography, Texas A\&M University, College Station, TX 77843, USA, Email: b-giese@tamu.edu

(10) e-Science Centre, ESSC (Environmental Systems Science Centre), Harry Pitt Bld, 3 Earley Gate, Reading University, Reading RG6 6AL United Kingdom, Email: bernd.giese@sci.monash.edu.au

(11) NOAA/PMEL/OCRD (National Oceanic and Atmospheric Administration/Pacific Marine Environment Laboratory/Office Chief of Research and Development), 7600 Sand Point Way NE Seattle,

WA 98125 USA, Email: D.E.Harrison@noaa.gov

${ }^{(12)}$ Massachusetts Institute of Technology, Department of Earth, Atmospheric and Planetary Sciences,

77 Massachusetts Avenue, Cambridge MA 02139 USA, Email: heimbach@mit.edu; cwunsch@mit.edu

${ }^{(13)}$ Meteorological Research Institute, Japan Meteorological Agency, 1-1 Nagamine, Tsukuba 305-0052, Japan, Email: mkamachi@mri-jma.go.jp

(14) Global Modeling and Assimilation Office, Code 610.1, NASA (National Aeronautics and Space Administration) Goddard Space Flight Center, Greenbelt, MD 20771, USA, Email: christian.keppenne@nasa.gov; michele.m.rienecker@nasa.gov

${ }^{(15)}$ Centro Euro-Mediterraneo per i Cambiamenti Climatici and Istituto Nazionale di Geofisica e Vulcanologia, Viale A. Moro 44, 40127 Bologna, ItalyEmail: masina@bo.ingv.it

${ }^{(16)}$ Atmospheric and Environmental Research, Inc., 131 Hartwell Avenue, Lexington, MA 02421-3126 USA, Email: rponte@aer.com

(17) NOAA (National Oceanic and Atmospheric Administration) Geophysics Fluid Dynamics, Princeton University, PO Box 308, Princeton NJ 08540, U.S.A., Email: Tony.Rosati@noaa.gov

${ }^{(18)}$ Alfred-Wegener-Institute for Polar and Marine Research, Postfach 1201 61, 27515 Bremerhaven, Germany Email: Jens.Schroeter@awi.de

${ }^{(19)}$ Met Office Hadley Centre, FitzRoy Road, Exeter, United Kingdom, Email: doug.smith@metoffice.gov.uk ${ }^{(20)}$ Centre Européen de Recherche et de Formation Avancée en Calcul Scientifique, 42 avenue Gaspard Coriolis, 31057 Toulouse, France, Email: weaver@cerfacs.fr

\begin{abstract}
Analyzing ocean variability, understanding its importance for the climate system, and quantifying its socio-economic impacts are among the primary motivations for obtaining ongoing global ocean observations. There are several possible approaches to address these tasks. One with much potential for future ocean information services and for climate predictions is called ocean synthesis, and is concerned with merging
\end{abstract}

all available ocean observations with the dynamics embedded in an ocean circulation model to obtain estimates of the changing ocean that are more accurate than either system alone can provide. The field of ocean synthesis has matured over the last decade. Several global ocean syntheses exist today and can be used to investigate key scientific questions, such as changes in sea level, heat content, or transports. This CWP (Community White Paper) summarizes climate variability as "seen" by several ocean syntheses, 
describes similarities and differences in these solutions and uses results to highlight developments necessary over the next decade to improve ocean products and services. It appears that multi-model ensemble approaches can be useful to obtain better estimates of the ocean. To make full use of such a system, though, one needs detailed error information not only about data and models, but also about the estimated states. Results show that estimates tend to cluster around methodologies and therefore are not necessarily independent from each other. Results also reveal the impact of a historically under-sampled ocean on estimates of inter-decadal variability in the ocean. To improve future estimates, we need not only to sustain the existing observing system but to extend it to include full-depth Argo-type measurements, enhanced information about boundary currents and transports through key regions, and to keep all important satellite sensors flying indefinitely, including altimetry, gravimetry and ice thickness, microwave SST (Sea Surface Temperature) observations, wind stress measurements and ocean color. We also need to maintain ocean state estimation as an integral part of the ocean observing and information system.

\section{INTRODUCTION}

The procedure of ocean state estimation provides an important tool for combining all available observations into a complete dynamical description of the timevarying ocean and its interaction with the remaining climate system. Results are especially useful for analyzing unobserved quantities, including the meridional overturning circulation, other transport properties, or air-sea interactions. Among others, such quantities are important information to be provided by ocean observing capabilities, particular for monitoring climate variations. In addition, the role of ocean state estimates in providing best possible present-day initial conditions for climate prediction systems is gaining attention and we can anticipate that in 10 years from now, ocean or coupled state estimations will be an integral component of an ocean and climate information and forecast system.

The vision of ocean state estimation as a means of bringing all ocean observations into a dynamically consistent description of the global time-varying ocean circulation goes back to the beginning of the "World Ocean Circulation Experiment" (WOCE) [1], although it took many years for its full implementation. Achieving this vision required a significant development of the in situ and satellite observing capabilities, together with improvement of ocean models and enhanced computational capabilities. Along the way, the picture of a steady ocean, that could be described by relatively simple dynamical principles as embedded in box models, evolved into one where the time variability has become the focus of interest and which requires a fully time-dependent and eddyresolving model as a dynamical basis. Important milestones in this development included the development of inverse methods (adjoint, Kalman filter, etc.) that can be applied to ocean circulation models using supercomputers. As an example, the development of ocean adjoint models encompassed the existence of modern primitive equation (PE) models (e.g. the MITgcm (MIT (Massachusetts Institute of Technology) global circulation model): [2]), the development of automatic differentiation tools [3], their pilot applications to ocean problems [4-6], and for that purpose, enhancements and adjustments of the computer infrastructure to finally encompass long optimization jobs. In the case of the ECCO (Estimating the Circulation and Climate of the Ocean) infrastructure, these accomplishments required close to a decade of sustained consortium efforts [5-6]. This development has proven to be a large endeavor that requires expertise in ocean observations, modeling, assimilation as well as information technology, which needs to be sustained and to have a long-term perspective to be effective.

Today, the products from several global and regional ocean data assimilation systems are available. Underlying assimilation schemes range from simple and computationally efficient (e.g. optimal interpolation) to sophisticated and computationally intensive (e.g. adjoint, Kalman filters, and smoothers). Some of the existing assimilation products span the period of the past several decades and are relatively coarse in spatial resolution; others focus on the data rich period, roughly from 1992 to present and tend to be eddy permitting, and sometimes only regional in extension. A summary of most of those efforts can be found in the CWP lead by [7]); it is specifically provided on the web page of CLIVAR's (Climate Variability and Predictability) "Global Synthesis and Observations Panel (GSOP), (http://www.clivar.org/organization/gsop/synthesis/synt hesis.php). Applications of those systems are expanding and already include many aspects of oceanography and climate research such as studies of sea level variability and changes (e.g. [5], [6], [8], [9], [10], [11] and [12]), water-mass analysis (e.g. [13-16]), mixed-layer heat balance (e.g. [17-19]). For instance, estimates of volume, heat, and freshwater transports of the global ocean have been obtained by fitting models to WOCE data (e.g. [6] and [20]). Many studies have also used various ocean reanalysis products to study the variations of the MOC (Meridional Overturning Circulation) in the Atlantic and its relations to heat transport and heat content changes. The relationship between atmospheric variability and a local or a remote response of the deep ocean was investigated by [21], and feedback processes acting during ENSO (El Niño/Southern Oscillation) were summarized by [22] and [8]) demonstrate the use of ocean state estimation for improving estimates of 
surface fluxes and [23-24] show applications of ocean state estimation for initializing coupled climate models. These are just examples, and the list could be made very long if we were trying to be complete.

Because underlying models and underlying assimilation approaches differ, results from individual ocean synthesis efforts also differ. But in the absence of formal error information it is difficult to judge where the truth resides and an important step toward improved estimates of the time-varying ocean state and its transport properties is therefore to understand the uncertainties in each estimate, before they can be combined into an improved ensemble estimate. This step is akin to what is common practice now in weather prediction and what is about to be adopted also for ensemble climate predictions. A pre-requisite to a respective multi-model ocean state estimation approach is a thorough inter-comparison of all available state estimates so as to understand the strengths and shortcomings in each and thereby to allow weighted ensemble means that are more accurate than any single product.

Respective work has started under the auspices of CLIVAR/GSOP's state estimation evaluation effort, which was co-sponsored by GODAE (Global Ocean Data Assimilation Experiment). Its objectives are to (1) examine the consistency of the syntheses (though multiproduct comparison), (2) evaluate the accuracy of these products (by comparison with observations), (3) estimate uncertainties, (4) identify areas where improvements are needed, (5) define the observational accuracies and requirements necessary to distinguish the quality of the syntheses and identify future observational requirements and (6) work on new approaches, such as coupled data assimilation. Many ocean reanalysis projects have participated in this effort that is based on many diagnostic quantities, including the comparison among reanalysis products and comparison with observations.

We will use results of the synthesis evaluation effort to identify improvements required to move toward an ocean information and forecast system for climate research and many other applications with socioeconomic benefits. However, results presented below are just examples, based on only a subset of the existing ocean state estimates listed by [7]. The selection principle was somewhat arbitrarily chosen to be a focus on estimates covering at least several decades in duration. Included in the inter-comparison were all available state estimates that started in 1980 or before and include efforts that use either dynamical or statistical models.

We observe from Fig. 1 a few important implications. Only z-level models are being used in long state estimates in the ocean and extending the spectrum to also include other vertical coordinates could be useful. At this point, state estimates in the ocean are based on five different primitive equation (PE) model runs with three different spatial resolutions. We also note that most of the attempts use similar data sets, but that major differences in the results could potentially be expected because of differences in the assimilation approaches and in the numerical details of the models. In particular, the way data are being used in the inversion procedure is important for determining the final solution, because the prior model and data error covariances have major impacts on the solution. The same holds for atmospheric reanalyses which show substantial difference in many aspects of the atmospheric state (see also CWP by [25])

The syntheses shown in Fig. 1 will be used here to demonstrate variability of only a few important climate variables, notably, (1) the oceans change in heat and freshwater content, (2) transports of heat and freshwater, and (3) changes in sea level. Results are intended to demonstrate the state of the field and lay the ground for a discussion of the future pathway. Complementary aspects of ocean state estimation are discussed in the CWPs of [7], [26] and [7].

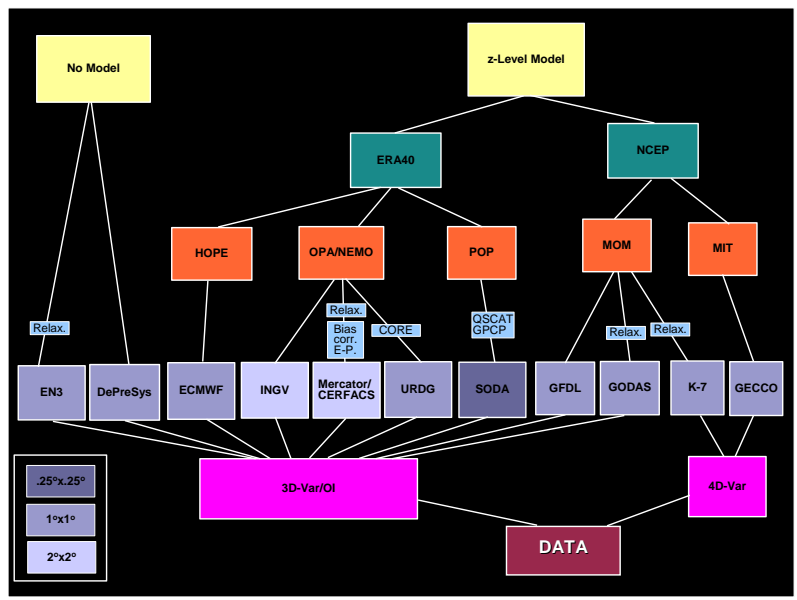

Figure 1. A summary of all syntheses included in this $C W P$, providing global data for at least 20 years. The efforts were sorted by assimilation approach (3D-Var

(3-Dimensional variational) and/or OI (Optimum Interpolation), or 4D-Var), by model type (HOPE (Hamburg Ocean Primitive Equation), OPA (Ocean PArallelise), POP (Parallel Ocean Program), MOM (Modular Ocean Model), and MITgcm), by atmospheric forcing (NCEP (National Centers for Environmental Prediction) and ERA4 (ECMWF (European Centre for Medium-Range Weather Forecasts) Re-Analysis)), and by spatial resolution. Also listed are additional features relevant to this analysis, such as relaxation in the surface layer, although these are not comprehensive and should indicate that these details are also important. 


\section{GLOBAL HEAT AND SALT CONTENT CHANGES}

Estimated changes of the heat content of the global ocean suggest an overall increase in the top $700 \mathrm{~m}$ during the last 50 years. As can be inferred from Fig. 2 (top panel), the increase is not monotonic and smooth but shows significant variations on all time scales. We can expect similar variability also to exist in future heat content changes (and all other climate variables for the same matter). A few other important observations from Fig. 2 are also noteworthy:

1) Most results show an increase in heat content in the 1970 s followed by a "cold period" in the late 1980s. There are only two estimates that do not follow this general pattern, including the 50-year long GECCO (German contribution to Estimating the Circulation and Climate of the Ocean estimate and the recent estimate from [28]. The latter is based entirely on ocean observations, but including a XBT (Expendable Bathythermograph) data set that was corrected for recently discovered errors arising from fall rate uncertainties [29].

2) All estimates showing the increase in heat content in the 1970s are based on 3D-Var or OI sequential approaches, which are strongly constrained to match the observations. As such they directly follow the data used as constraints. In contrast, the dynamically self-consistent GECCO solution did reject the heat content increase in the 1970s as being dynamically inconsistent and thus identified that period as one with enhanced data errors. This notion is now supported by recent studies using corrected XBT observations, although the picture given by [28] is likely to change again as the quality of XBT data might continue to improve. We note that adjoint models seem to provide an extra benefit - besides producing dynamically consistent estimate - that dynamics and first principles help to identify problems with observations.

3) During the 1990s we see a general increase in heat content; however the bulk of the estimates indicate a drop in heat content after 2004. We now know that this period was characterized by errors in the Argo data propagating into the heat content estimate of sequential estimates and that the state of the observational accuracy during this period is still evolving.

4) Finally, we note the large spread in all results toward the end of the time series, although mostly caused by the two shorter syntheses. This is counterintuitive given the fact that this period is best observed. We will see below that this finding propagates through many of the diagnosed quantities, pointing to a large sensitivity of the estimation on the underlying estimation methodology.

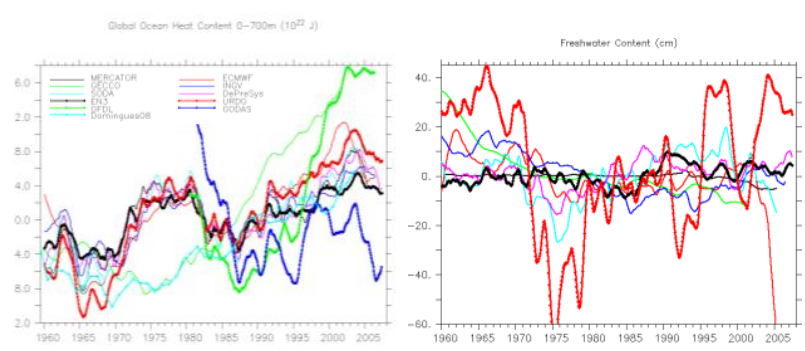

Figure 2. Global heat (top panel) and freshwater (bottom panel) content changes

Turning toward the global freshwater content of the ocean (Fig. 2b), the variability between all estimates is very large, highlighting the fact that the ocean was historically under-sampled, especially for salinity, and demonstrating a general problem of existing ocean simulations in determining the freshwater content from observations. This also affects estimates of many climate indices, including global sea level rise. Even now we are still lacking important information of temperature and salinity changes below the nominal Argo sampling depth of about $2000 \mathrm{~m}$. As a result, estimates of the ocean's freshwater content are plagued by large uncertainties and differences in the methodology may cause artificial signals, dependent on the details of the assimilation approaches. For instance, the large drop in 2004 visible in the ECMWF (European Centre for Medium-Range Weather Forecasts) ReAnalysis) product is related to the transition from the delayed mode altimeter maps to the real time altimeter maps, which had different global means. We also note that some of the implied variability of the freshwater content does mirror that of the heat content in the 1970s, suggesting ${ }^{\text {stathat }}$ the often used approach of constraining the ocean by artificial salinity derived from a climatological $\mathrm{T} / \mathrm{S}$ relation does lead to problems in a world of changing heat content (artificial or real) in the ocean. Finally, some estimates show a smooth decrease in freshwater content. Among those is the GECCO result. Several findings indicate that this decrease must be artificial, as the eustatic contribution to the sea level rise is believed to be positive throughout the last decades (e.g. [30]), and contributions increase during more recent years [31].

While global diagnostics of climate changes in the ocean are important, it is usually the regional changes that are of largest consequence and therefore of major interest. Since the major basins were quite differently sampled over the last decades the different levels of agreement between the syntheses in the different basins may provide information about the influence of different observing systems. We show in Fig. 3 changes in the heat content, but now diagnosed (a) in the North Atlantic, (b) the North Pacific, (c) the Indian Ocean, and (d) the Southern Ocean. Again, all results represent 
changes in the heat content in the top $700 \mathrm{~m}$. Most noticeable is the good agreement in the North Atlantic Ocean supported by the fact that this is the bestobserved basin. The North Pacific shows again a large consistency between all sequential estimates. But it is especially here where GECCO shows a decrease in the heat content in the 1970s and a substantial increase subsequently. An inspection of GECCO results in all panels suggest that a heat content minimum first occurred in the Southern Ocean in the late 1960s/early 1970 s followed by a rapid increase in heat content, that leveled off in the early 80s. A similar behavior can be seen in the North Pacific and the Indian Ocean, but shifted in time. The figure also suggests a decrease in heat content in the North Pacific since about 2000, in the North Atlantic a respective decrease could be inferred only after 2004. Finally we see a larger spread in all sequential estimates in the Southern Ocean and the Indian Ocean, reflecting the fundamental undersampling of both basins. This under-sampling lasted until recently and is responsible for the increased spread of global estimates toward the end of the considered period.
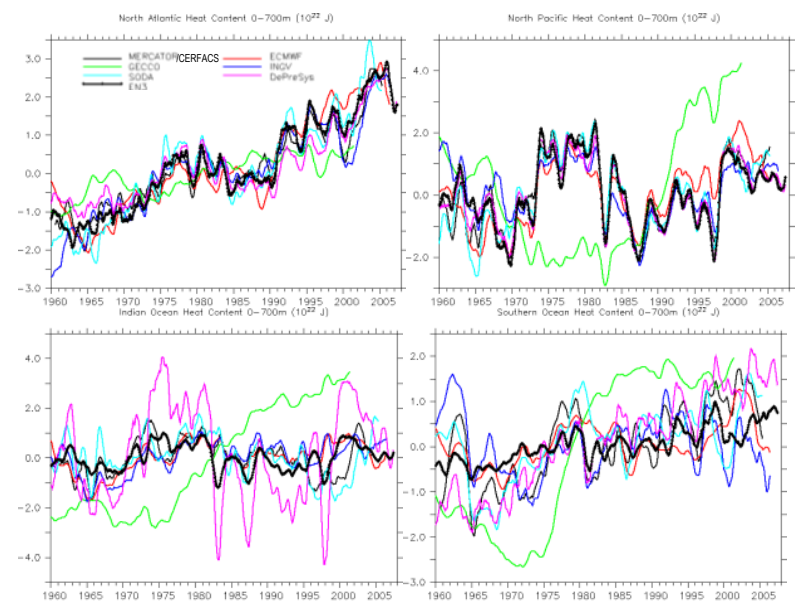

Figure 3. Heat Content time series in several ocean basins.

\section{HEAT AND SALT TRANSPORTS}

Among the important quantities that need to be diagnosed from ocean synthesis are transport of properties like heat and freshwater, $\mathrm{CO}_{2}$, nutrients, oxygen and many other substances. To provide a few examples, we show in Fig. 4 transports of heat and freshwater across $48^{\circ} \mathrm{N}$ and $25^{\circ} \mathrm{N}$ in the North Atlantic, across the equatorial Pacific, and across $10^{\circ} \mathrm{S}$ in the Indian Ocean. It is obvious from the figure that, while integral quantities such as the heat content appear consistent in the North Atlantic, estimates of transport properties at specific latitudes do not necessarily agree, leading to a large spread of results in the Atlantic which is by no means smaller than in all other basins. This is not surprising since the estimated transport properties are most sensitive to details of the assimilation approaches and they may be especially sensitive to dynamical inconsistencies introduced during the process of merging data and models. The highest consistency appears in the tropical Pacific, where at least the phase (but not the amplitude) of heat transport variations agrees among all results, although freshwater transports do show an increased spread. All other time series show only small commonalities.
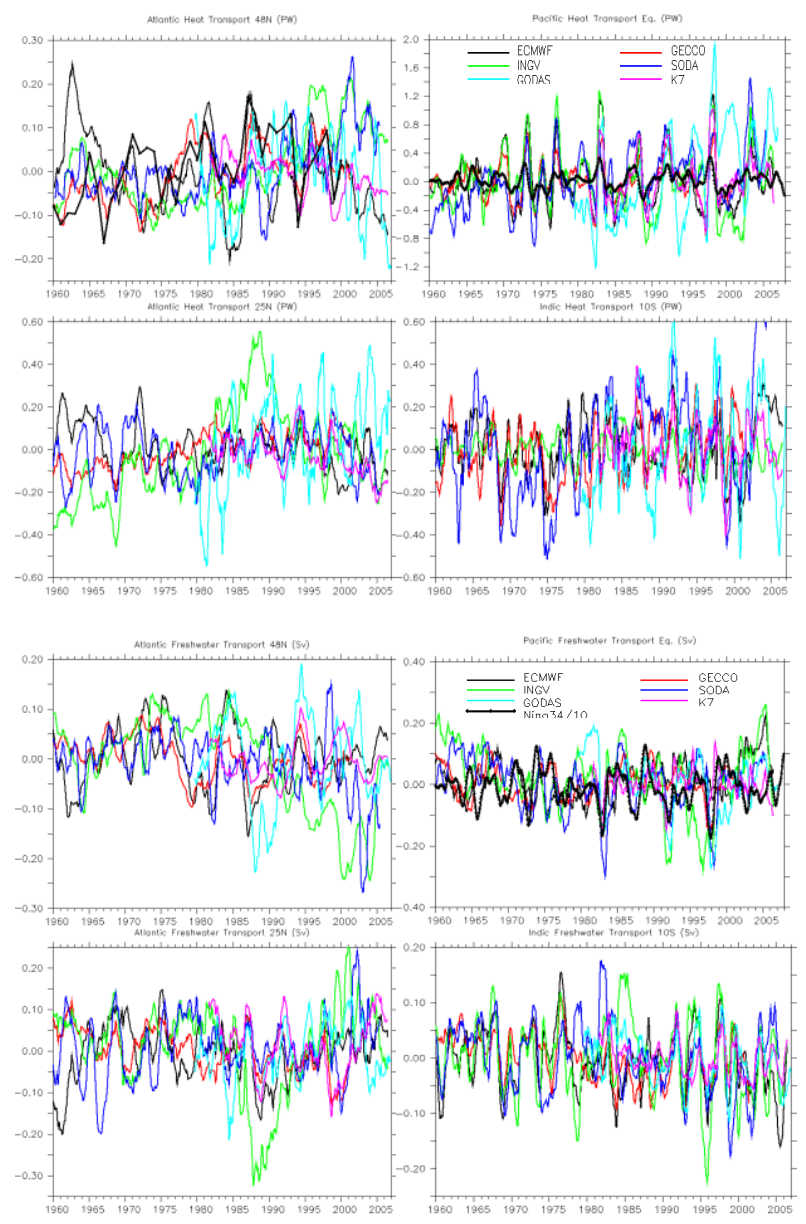

Figure 4. Heat and freshwater transports.

\section{GLOBAL AND REGIONAL SEA LEVEL CHANGES}

A quantity of general concern is sea level and its variability, which represents an integral over many individual aspects of the ocean state. Changes in sea level potentially can have a substantial impact on society and understanding ongoing and past changes and their regional character is therefore of specific importance. We show in Fig. 5 estimates of global sea level changes as they follow separately from the thermo- and halosteric anomalies in several ocean state estimates. Not surprisingly, both panels reflect essentially the previously shown changes in heat and salt/freshwater content. However, it is noteworthy that the three estimates (GECCO, ECMWF, and 
Mercator/CERFACS), showing a significant thermosteric increase during the 1990s, make significant direct use of altimetry.

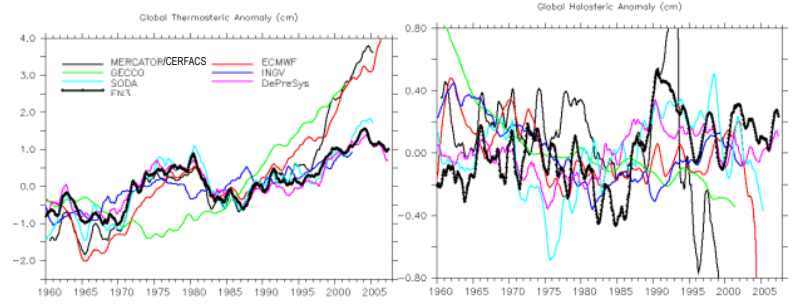

Figure 5. Global steric surface height anomalies

Turning again to regional changes, Fig. 6 shows estimates of local thermosteric and halosteric SSH (Sea Surface Height) changes as they follow from three estimates representing the spread that exists from all available results. On the one end, SODA (Simple Ocean Data Assimilation) represents those results that use in situ profiles to correct the model's T/S structure locally in space and time. In those approaches, altimetry is projected on synthetic T/S changes and only the latter are used as constraints. The other end of the spectrum is represented by the adjoint-family of approaches, encompassing all ECCO results, but also K7 from Japan. As an intermediate group we show also results from ECMWF, which is tuned to improve SI forecasts. Results are shown for the periods 1962-2001 and 1992 2001, respectively.

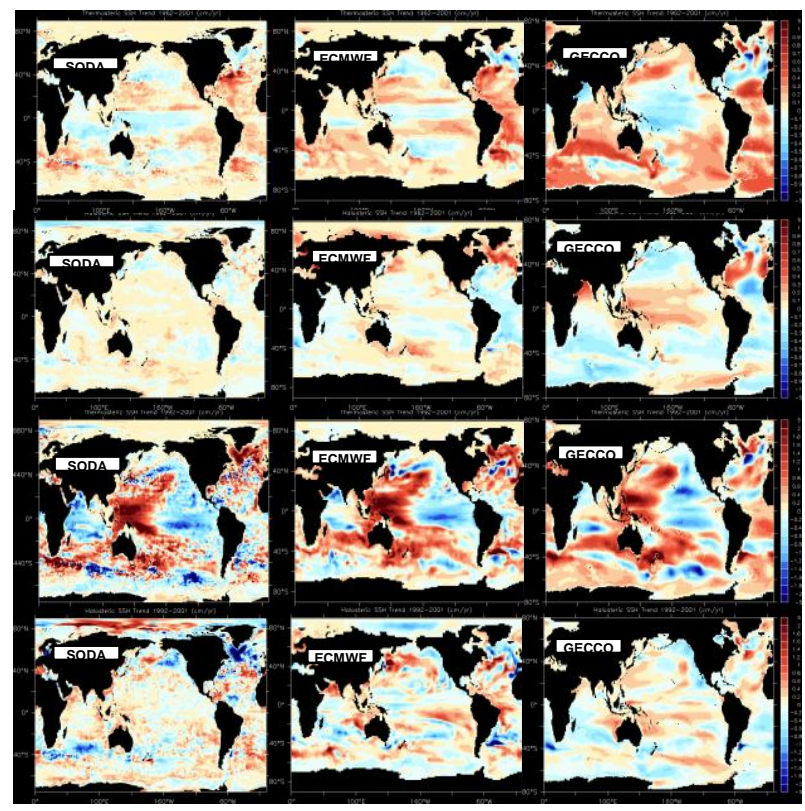

Figure 6. Estimates of local thermosteric and halosteric SSH trends as they follow from three estimates representing the spread that exists from all available results. Top two rows represent the period $1962-2001$; the bottom two rows represent the period $1992-2001$.

Starting with the first period (top two rows of the figure) we realize that estimated trends differ substantially in all three examples, especially between SODA and GECCO. This is obvious for temperature but holds equally well for salinity. Over large parts of the world ocean, GECCO results suggest that $\mathrm{SSH}$ changes induced by heat content changes and changes in salinity counterbalance to some extent as this would follow from either advection of water masses along isopycnals (e.g. wind- driven changes in GECCO) or imposed by the use of $\mathrm{T} / \mathrm{S}$ relations. A respective counterbalance is less visible in the SODA result that contains signatures of eddy noise. The latter aspect is even more obvious in the more recent years characterized by the availability of altimeter data that can partly compensate for the lack of resolution. Nevertheless, large-scale trends in the thermosteric SSH now agree in all three estimates. Halosteric estimates also tend to converge but there remain substantial differences, e.g. in the subpolar North Atlantic. We also note that Boussinesq model systems cannot account for a non-conservative global ocean mass increase as is being observed by the altimeters (real ocean mass is growing due to land-ice melt). This has to be accounted for during the assimilation process by removing a global trend in the data.

\section{UNCERTAINTIES IN OCEAN STATE ESTIMATES}

A major issue with all data assimilation products currently available is that no formal estimates of uncertainties of the estimated states or derived information are provided. Primarily this is because computing error information may be costly and often not tractable (even on the largest computers available) given the large dimension of the problem, although no fundamental (mathematical) obstacle exists to do so. In particular for adjoint methods like ECCO/GECCO and $\mathrm{K} 7$ the error estimation process is rather costly as it involves the computation of the inverse of the Hessian [32]. However, the accuracy of these formal error covariance estimates is highly dependent on the accuracy of the error covariance estimates of the input fields and these are subject to considerable uncertainty. Estimating uncertainties of existing ocean state estimates therefore remains a major challenge. In addition to the three dimensional estimations of the ocean state at a given time (analysis problem), an uncertainty information of the time evolution is also required in a reanalysis, which will be sensitive to the time variations of the observing system, to the errors of the ocean model, of the atmospheric fluxes, and of the assimilation system, which are often flow-dependent and not easy to estimate.

Sequential methods such as the Kalman Filter actually provide posterior error estimates as part of the analysis and could start to provide this information along with their estimate. However, current implementations rely 
on low rank approximations of uncertainties, which are also obtained during the optimization process in the adjoint method and it is not clear whether they are able to provide useful information. Reference [33] provides an ocean application for a relatively short period of 14 days. Moreover, the analysis error provides only one part (the aleatoric part) of the error. The other part, the epistemic error related to the biases of the individual estimates, may dominate the error rendering the estimation of the first error obsolete. As in atmospheric applications, ensemble methods can help as a first guess of the estimation uncertainty, in a way similar to multimodel forecasts. An ensemble spread of different reanalysis products can be used to get a first estimate of the time evolution and uncertainty of the climate reconstructions, although any caveat of such an ensemble methodology should be taken into account when interpreting the results. This approach was used by [34] in the comparison of ocean reanalyses organized by the CLIVAR GSOP. Using the results presented above, it is now tempting to specify especially large uncertainties in estimated transports.

The issue related to the computation of formal error bars for state estimation results arises in part because data assimilation schemes usually search for circulation estimates in the full space of the model. If searches are performed in the space spanned by only the observations instead, the expected analysis errors are more readily available. So some of the shortcomings of current ocean data assimilation products appear to stem from the approaches used. Significant efforts are required in the future to improve our way of performing optimizations, and it can be expected that in many cases there are better ways of performing optimizations. This does include expanding the parameter space so that model physics are improved and thereby ocean processes are better represented (e.g. overflow processes and water mass formation or conservation). Finally, ways of estimating initial conditions for the optimized state also need to be developed so as to minimize numerical adjustments during the first years of the runs, which currently plague many available products.

Ideally each synthesis should come with an estimate of the analysis error and much needs to be done over the next years to provide progress in this specific direction. The error would make estimated ocean states and derived information much more valuable. It would also allow computing an ensemble synthesis with a further reduced error under the assumption that the estimates are bias-free. Assuming for the moment that all estimation biases are random, independent and of similar amplitude (certainly, as described in the introduction, these conditions are not likely to hold), an effort can be made to obtain improved transport estimates by averaging all available results along with an error estimate that includes both of the above described components.

Results are shown in Fig. 7, which display the ensemble mean as well as the ensemble spread of temperature. From an inter-comparison of time series of heat and freshwater transports, large climate excursions are obvious, as we would expect from NAO, ENSO or other such phenomena. We also observe an anti-correlation of heat and freshwater transports suggesting that the net effect, e.g. on density, is smaller than it would follow from just one component. Again, observing and estimating all components of the ocean state are required for quantitative applications. In all panels we see the tendency to an increased spread toward the end of the estimate period, suggesting that solutions during the earlier years are mostly determined by the surface forcing and perhaps similar initial conditions, while data impacts and the details of underlying assimilation procedures are especially large toward the end. This could suggest that at least some of the assimilation results are more biased than others and do not make maximum use of the data and the dynamics embedded in the model. This result could suggest that the relatively good agreement in the early years just reflects common biases in all estimates.

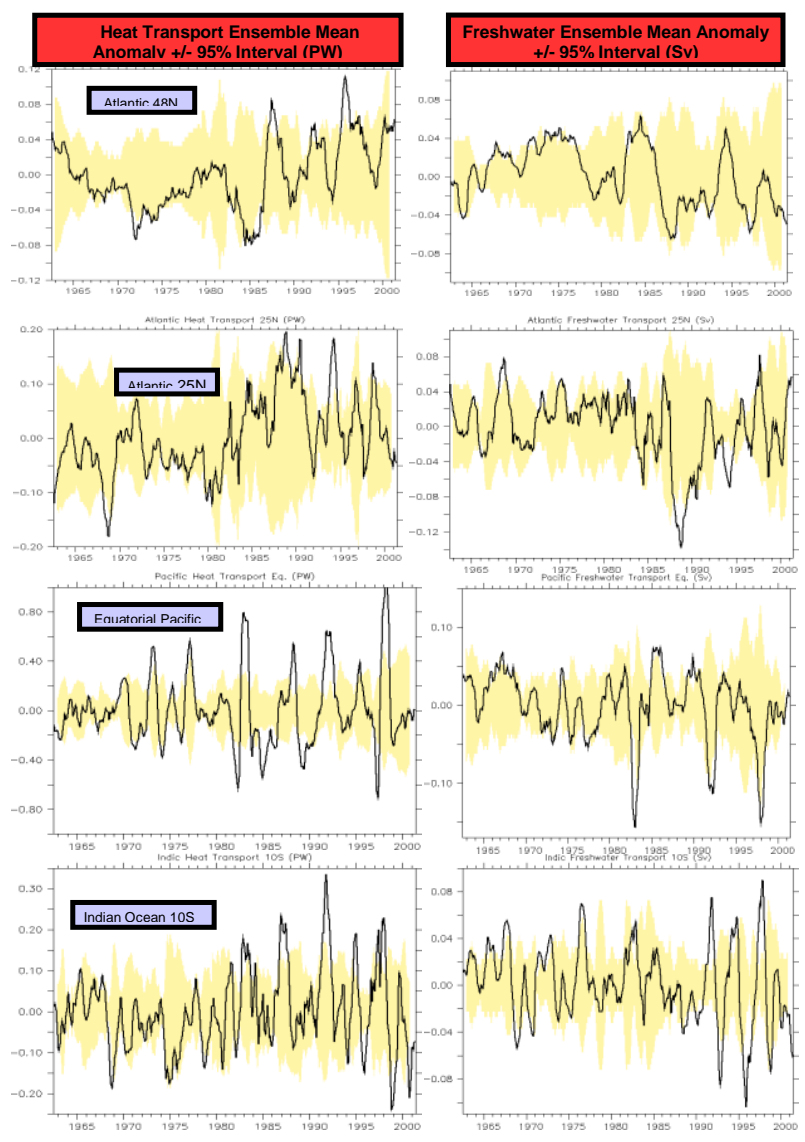

Figure 7. Ensemble mean of transports plus uncertainties. 
Two questions could now be raised that should prevent us from being either overly optimistic if results agree or from being overly pessimistic if they do not:

-Does agreement among several solutions have anything to do with confidence in the results and do ensemble averages provide better approximations to the truth? This is a necessary condition since there is only one truth. In order to apply this as a sufficient condition the results need to be randomly distributed around the truth as indicated in Fig. 8a. In this case ensemble averages are better approximations to the truth and the deviation to the ensemble mean serves as a means to assess a particular synthesis. Moreover, assimilation is expected to move each solution towards the truth, which will cause a reduction in ensemble variance that can then be used to assess the success of the assimilation. However, we can see from Fig. 1 that syntheses based on common approaches lead to similar results. Figure 2 nicely demonstrates this feature, and one has to carefully evaluate whether the estimates can be regarded as independent before conclusions about uncertainty are drawn.
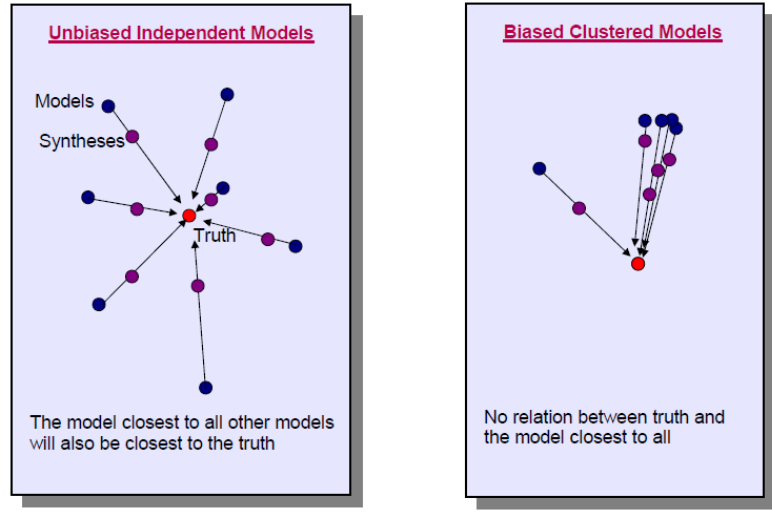

Figure 8. Evaluating model quantity from multiensemble of results. The arrows illustrate the general expectance that assimilation of observations moves the results closer to the truth. The left panel show the ideal situation in which the ensemble spread and the distance to the ensemble mean provide useful measures while the

right panel illustrates a biased case that is more

realistic for the ensemble of present day synthesis.

- Do we expect that data constraints will bring different models closer together and thus closer to the truth? The latter again is a necessary condition - at least for the assimilated data. However, results based on the same underlying model employing different data and/or data assimilation schemes may diverge. In this case, as illustrated in Fig. 8b, the assimilation process may increase the ensemble spread and neither the spread nor the closeness to the ensemble mean have any significance and one has to be careful not to expect a greater agreement among syntheses than for pure simulations. A comparison with the results from the corresponding reference experiments (without data assimilation) could provide further insight in the present results, although this was not yet attempted. The largest challenge for future synthesis products will be to provide realistic error bars that are consistent with independent data.

\section{THE WAY AHEAD}

Ocean state estimation was developed over the last decade, envisioning that it will become a firm element of an ocean observing and information system, providing the best possible description of the time evolution of the ocean after merging available ocean observations with the dynamics embedded in ocean circulation models. Today this goal has been reached in a prototype fashion, although many problems remain which require specific attention during the next decade. Meanwhile, a suite of regional and global ocean synthesis products is already available with varying horizontal resolutions, extending up to 50 years in duration. The number of studies using these products for oceanographic and climate-related studies already covers a wide range of topics, including the initialization of coupled climate models, and the computation of climate indices. For the years to come it will be essential for the community to recognize the value of ocean state estimation and to expand their applications of ocean synthesis products for research and information services alike.

Ongoing synthesis comparison efforts reveal a large spread in some of the results, especially in terms of ocean transport. Quite clearly, the spread to some extent is due to different approaches, underlying data sets, or differing control terms. However, we also note that the spread, at least in terms of heat and freshwater content, increases toward the end of the data record characterized by the largest number of observations. This is counter-intuitive, suggesting that the shown syntheses moved further away from reality toward the end of the runs. The situation will be different in shorter runs covering only the period after about 1990, however, there will be other problems, e.g. those related to initial numerical drifts. Since the spread toward the end of the assimilation period is induced by the assimilated data (representing the truth), an optimistic expectation would be that the obtained states are for these last years more randomly distributed around the truth for these last years. If that is true, ensemble means would provide better estimates which are less prone to common biases.

To increase the value of ocean state estimation products, much effort is needed over the next decade to characterize the uncertainties in each synthesis product and to improve them through advanced assimilation approaches. The outcome should be a merged, 
weighted, ensemble mean to produce better estimates and reduced uncertainties relative to what can be obtained from one approach alone. A concerted comparison effort based on runs with the same data, period, etc., and expanded analysis looking at controls+analyses+innovations to understand existing differences and to learn how to improve results seems to be one of the important next steps. At the same time formal uncertainties for each estimate are required, which, once available, would quantify the quality of each, at least according to its assumed statistical inputs.

Until formal error bars are provided, and shown to be reliable, we can only speculate as to why we obtain differences between synthesis approaches. Supported through the division into two clusters, it appears that the assimilation approach itself does have a substantial impact on the result, probably more so than slight differences in the constraining data. In particular, we see a clear separation of results clustered into adjoint smoother estimates and filter approaches. Both make different use of the data and the underlying model dynamics and as can be seen from Fig. 6, filter approaches lead to more noisy results and appear to be more slaved by individual data than are smoothers using all data over a long period. The effect is that in filterapproaches the insertion of new data leads to local unbalanced anomalies which set of adjustment processes in the form of planetary waves, which subsequently imprint themselves on integral quantities like heat transports. All model results shown here have roughly the same resolution but use data differently (comparing results with a differing resolution we do anticipate similar spreads in the solution as we see it from process-oriented forward modeling). Because of this ocean processes are also represented or corrected differently. As an example, the insertion of temperature and salinity in filter approaches maintains water masses that are being created, e.g. through mixing processes up stream. In contrast, smoother efforts do not insert data but attempt to have the model simulate them through changes in control parameters, such as surface forcing. This implies that missing physics like mixing needs to be compensated for through changes in surface forcing. Yet smoother methods provide the opportunity to include mixing and water mass formation as a control parameter and thereby to improve the model physics through parameter estimation. This process will allow improvements in overflow and mixing processes during the assimilation effort in the future.

But in general terms, ocean state estimation products can only be as good as the data provided and at the same time require best possible model representation. State estimation efforts therefore need to be tightly coupled to model development and improvement efforts as described in the CWP by [35]. We envision especially that the field of ocean state estimation will move toward coupled assimilation efforts. First pilot applications exist today and several others are in the process of spinning up. The move towards coupled state estimation activities will lead to improved ocean information for coupled forecasts ranging from near-term to seasonal to decadal time scales. It should also be acknowledged that there is a need for regional synthesis efforts also using high resolution regional models, high resolution and high quality atmospheric forcing products, good quality bathymetric data, etc., since many of the socioeconomic problems associated with climate variability and climate change will be manifested at the regional scale.

At the same time, ocean state estimation has a deep need for high-quality ocean data, especially that covering the decades before the 1990s and possibly going back to the beginning of the century. Recent issues around biases and quality control highlight this need and community efforts need to be stepped up around this issue. This quality-control process can build firmly on the estimation process itself as was shown by the GECCO results (e.g. [36]. It is are already part of the AGRO quality control system [37]. In fact, residuals from dynamically consistent estimation approaches are a valuable result that needs to be evaluated with respect to uncertainties in observations and models alike. At the same time estimation approaches need to be used much more in the future to guide the evolution of the observing system than it was done in the past (see also the CWP by [27]. As mentioned above, having full depth hydrographic information in the open ocean and under sea ice appears a very important step towards improved estimates of sea level, but also of the hydrological cycle. Those data sets should be complemented by boundary current transport information, e.g. like the Florida Current. The consequence of the failure of various models to properly represent the vertical structure of the overturning [38] for climate studies is yet unknown and we can logically expect them to be significant on decadal timescales. However, it remains to be tested if density information directly at the boundary will improve the estimates of MOC above what a broad-scale observing system provides, including satellite surface height information.

Anticipating further improvements, we expect that over the decade following OceanObs'09 ocean state estimates will be an essential part of the infrastructure that will provide information about the time-varying ocean on a regular basis and for many scientific applications and climate services. As part of this, we envision a regular evaluation of the ocean state over the full water column, providing information about important ocean indices to the community at large on a regular basis. Furthermore, we expect ocean state estimation to become an important part of seamless climate predictions, including seasonal, interannual and 
decadal timescales. Efforts for the longer time scale have started under the IPCC umbrella, but much has to be learned about the best use of ocean data and ocean information before forecasts become quantitative. It can also be expected that best forecasts will be produced by coupled models that can be directly constrained by climate data (coupled data assimilation). Regardless of what the application will be, we need to spend significant effort to improve the quality of ocean state estimation products. This will involve an improved use of existing data by incorporating full error covariances about data and models. Such information does not exist currently, and we expect as part of an evolution of the observing system to include more information about uncertainties of observations.

Essentially, after a decade of development, the community is now ready to use ocean state estimation tools on a routine basis as part of an observing strategy to extract information out of the existing observing system, and to help to improve the observing system so as to maximize the amount of information that can be extracted. To do this in a most efficient way, a longterm ocean synthesis strategy is required, similar to what is ongoing in the atmospheric reanalysis community. The benefit of such a long-term perspective will be that an expertise and an infrastructure can be built up and maintained. Observations are the essential input for ocean state estimation. However, we should think of ocean state estimation as an integral part of the observing strategy, not a customer, capable of delivering important information about the changing ocean state.

\section{REFERENCES}

1. Munk, W. \& Wunsch C. (1982). Observing the Ocean in the 1990s. Phil. Trans. R. Soc. Lond., A307, 439-464.

2. Marshall, J., Adcroft A., Hill C., Perelman L. \& Heisey C. (1997). A finite-volume, incompressible Navier-Stokes model for studies of the ocean on parallel computers, $J$. Geophys. Res., 102, 5753-5766.

3. Giering, R. \& Kaminski T. (1998). Recipes for adjoint code construction. ACM Trans. on Math. Software, 24, 437474.

4. Marotzke, J., Giering R., Zhang Q. K., Stammer D., Hill C. N. \& Lee T. (1999). Construction of the adjoint MIT ocean general circulation model and application to Atlantic heat transport sensitivity. J. Geophys. Research, $104,29,529-29,548$.

5. Stammer, D., Wunsch C., Giering R., Eckert C., Heimbach P., Marotzke J., Adcroft A., Hill C.N. \& Marshall J. (2002a). The global ocean circulation during 1992 -1997, estimated from ocean observations and a general circulation model. J. Geophys. Res., 107(C9), 3118, doi:10.1029/2001JC000888.

6. Stammer, D., Wunsch C., Fukumori I. \& Marshall J. (2002b). State estimation improves prospects for ocean research. EOS, Transactions, American Geophysical Union, Volume 83, Nr. 27, p. 289, 294--295.

7. Lee, T. \& Co-Authors (2010). "Ocean State Estimation for Climate Research" in these proceedings (Vol. 2), doi:10.5270/OceanObs09.cwp.55

8. Stammer, D., Ueyoshi K., Köhl A., Large W.B., Josey S. \& Wunsch C. (2004). Estimating Air-Sea Fluxes of Heat, Freshwater and Momentum Through Global Ocean Data Assimilation. J. Geophys. Res., 109, C05023, doi:10.1029/2003JC002082.

9. Carton, J.A. \& Giese B.S. (2008). A reanalysis of ocean climate using Simple Ocean Data Assimilation (SODA). Mon. Wea. Rev., 136, 2999-3017.

10. Wunsch, C., Ponte R.M. \& Heimbach P. (2007). Decadal trends in sea level patterns: 1993-2004. J. Clim., 20, 5889-5911.

11. Köhl, A. \& Stammer D. (2008a). Variability of the meridional overturning in the North Atlantic from 50year GECCO state estimation. J. Phys. Oceanogr., 38, $1913-1930$

12. Köhl, A. \& Stammer D. (2008b). Decadal sea level changes in the 50-year GECCO ocean synthesis. $J$. Clim., 21, 1866-1890.

13. Fukumori, I., Lee T., Cheng B. \& Menemenlis D. (2004). The origin, pathway $\&$ destination of NINO3 water estimated by a simulated passive tracer and its adjoint. J. Phys. Oceanogr., 34, 582-604.

14. Wang, O., Fukumori I., Lee T. \& Cheng B. (2004). On the cause of eastern equatorial Pacific Ocean T-S variations associated with El Nino. Geophys. Res. Lett., 31, L15310, doi:10.1029/2004GL020188.

15. Masuda, S., Awaji T., Sugiura N., Toyoda T., Ishikawa Y. $\&$ Horiuchi K. (2006). Interannual variability of temperature inversions in the subarctic North Pacific, Geophys. Res. Lett., 33, doi:10.1029/2006GL027865.

16. Toyoda, T., Awaji T., Masuda S., Sugiura N., Igarashi H., Mochizuki T. \& Ishikawa Y. (2009). Interannual variability of North Pacific eastern subtropical mode water formation in the 1990s derived from a 4dimensional variational ocean data assimilation experiment. (submitted to J. Geophys. Res.).

17. Kim, S.-B., Lee T. \& Fukumori I. (2004). The 1997-99 abrupt change of the upper ocean temperature in the northcentral Pacific. Geophys. Res. Lett., 31, L22304, doi:10.1029/2004GL021142.

18 Kim, S.-B., Lee T. \& Fukumori I. (2007). Mechanisms controlling the interannual variation of mixed layer temperature averaged over the NINO3 region. J. Clim, 20, 3822-3843.

19. Halkides, D. J., and T. Lee (2009), Mechanisms controlling seasonal-to-interannual mixed layer temperature variability in the southeastern tropical Indian Ocean, J. Geophys. Res., 114, C02012, doi:10.1029/2008JC004949.

20. Stammer, D., Wunsch C. \& Giering R., et al. (2003). Volume, heat, and freshwater transports of the global 
ocean circulation 1993-2000, estimated from a general circulation model constrained by World Ocean Circulation Experiment (WOCE) data/ J. Geophys. Res.Oceans, 108,

21. Masina S., Di Pietro P. \& Navarra A. (2004). Interannualto-decadal variability of the North Atlantic from an ocean data assimilation system. Climate Dynamics, 23, 531-546, doi:10.1007/s00382-004-0453-6.

22. Capotondi, A., Wittenberg A. \& Masina S. (2006). Spatial and temporal structure of ENSO in $20^{\text {th }}$ century coupled simulations. Ocean Modelling, 15, (3-4), 274-298.

23. Pierce, D. W., Barnett T. P., Tokmakian R., Semtner A., Maltrud M., Lynsey J. \& Craig A. (2004). The ACPI project, element 1: Initializing a coupled climate model from observed initial conditions. Clim. Change, 62, 1328.

24. Pohlmann, H., Jungclaus J., Köhl A., Stammer D. \& Marotzke J., (2009). Initializing Decadal Climate Predictions with the GECCO Oceanic Synthesis: Effects on the North Atlantic. J. Climate, 22, 3926-3938. doi:10.1175/2009JCLI2535.1.

25. Trenberth, K. \& Co-Authors (2010). "Atmospheric Reanalyses: A Major Resource for Ocean Product Development and Modeling" in these proceedings (Vol. 2), doi:10.5270/OceanObs09.cwp.90

26. Balmaseda, M. \& Co-Authors (2010). "Initialization for Seasonal and Decadal Forecasts" in these proceedings (Vol. 2), doi:10.5270/OceanObs09.cwp.02

27 Heimbach, P. \& Co-Authors (2010). "Observational Requirements for Global-Scale Ocean Climate Analysis: Lessons from Ocean State Estimation" in these proceedings (Vol. 2), doi:10.5270/OceanObs09.cwp.42

28. Domingues, C. M., Church J. A., White N. J., Gleckler P. J., Wijffels S. E., Barker P. M. \& Dunn J. R. (2008). Improved estimates of upper-ocean warming and multidecadal sea-level rise. Nature, 453, 1090-1093.

29. Wijffels, S. E., Willis J., Domingues C. M., Barker P., White N. J., Gronell A., Ridgeway K., Church J. A. (2008). Changing eXpendable BathyThermograph fallrates and their impact on estimates of thermosteric sea level rise. J. Clim., 21, 5657-5672.

30. Munk W. (2003). Ocean freshening, sea level rising. Science, 300, 2041-2043.

31. Cazenave, A., Dominh K., Guinehut S., Berthier E., Llovel W., Ramillien G., Ablain M. \& Larnicol G. (2009). Sea level budget over 2003-2008: A reevaluation from GRACE space gravimetry, satellite altimetry and Argo. Global and Planetary Change.

32.Fisher M. \& Courtier P. (1995, Estimating the covariance matrix of analysis and forecast error in variational data assimilation. ECMWF Technical Memorandum No. 220.

33. Powell, B. \& Moor A.M. (2009), Estimating the 4 DVAR analysis error of GODAE products. Ocean Dynamics (2009) 59:121-138 doi:10.1007/s10236-008-0172-3

34.Balmaseda, M.A. \& Weaver A. (2006). Temperature, salinity, and sea-level changes: climate variability from ocean reanalyses.

http://www.clivar.org/organization/gsop/synthesis/synth esis.php.

35. Griffies, S. \& Co-Authors (2010). "Problems and Prospects in Large-Scale Ocean Circulation Models" in these proceedings (Vol. 2), doi:10.5270/OceanObs09.cwp.38

36. Liu, H (2009). Sensitivity of warming trends in instrumental biases of XBT's. Diploma Thesis, University of Hamburg, pp. 80.

37. Gaillard, F., Autret E., Thierry V., Galaup P., Coatanoan C. \& Loubrieu T. (2009). Quality control of large Argo data sets. J. Atmos. Ocean. Tech., 26, 337-351.

38. Saunders, P. M., Cunningham S. A.,, de Cuevas B. \& Coward A. C. (2009). Decadal changes in the North Atlantic and Pacific meridional overturning circulation and heat flux. J. Phys. Oceanogr., 38, 2104-2107. 